\title{
Periostales Chondrosarkom des Zungenbeins
}

\author{
B. Lehnert' ${ }^{1}$, R. Weiß ${ }^{1}$, U. Stein ${ }^{2}$, T. Verse ${ }^{1}$
}

1 Universitätsklinik und Poliklinik für Hals-Nasen-Ohrenheilkunde, Homburg/Saar (Komm. Direktor: PD Dr. Th. Verse)

${ }^{2}$ Institut für Pathologie der Universitätsklinik des Saarlandes, Homburg/Saar (Direktor Prof. Dr. Remberger)

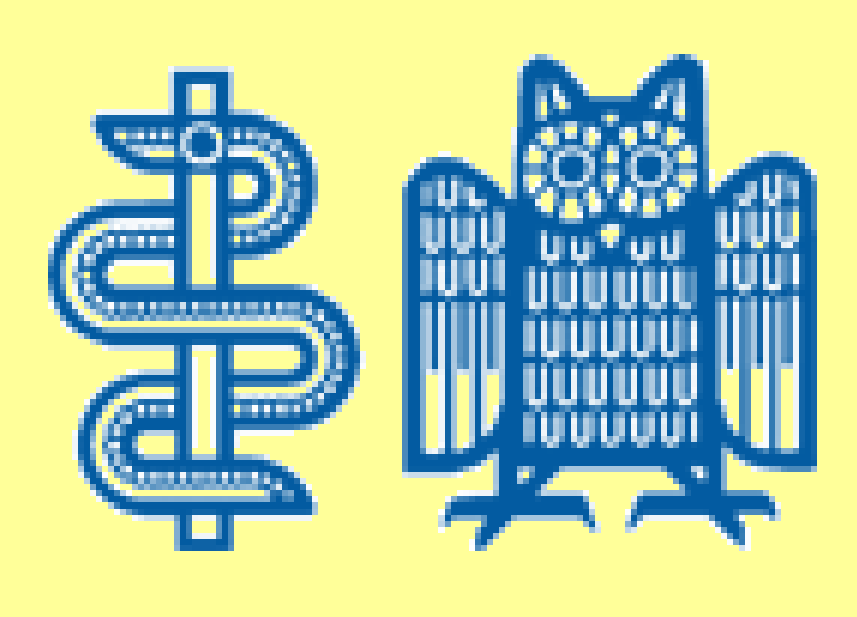

\section{Chondrosarkome im Kopf-Hals-Gebiet}

Etwa 0,1\% aller Kopf-Hals-Malignome sind Chondrosarkome. Chondrosarkome sind maligne Tumoren, die Chondroid (atypisches Knorpelgewebe) aber -in Abgrenzung zum Osteosarkom- kein Osteoid bilden. Sie gehen häufiger von Knochen als von Knorpel aus. Betroffen sind in der Regel Becken, Femur, Humerus und Rippen. ${ }^{1,3}$ Die 5-JÜR von Chondrosarkomen aller Körperregionen wurden mit $90 \%$ für G I, $81 \%$ für G II und $43 \%$ für G III- Tumoren angegeben².

Chondrosarkome des Halses entstehen z. B. am Larynx, wo sie zu 70\% dem Schild- und zu etwa 30\% dem Ringknorpel entspringen. Sind die häufigsten Sarkome des Larynx.

Das Zungenbein ist eine sehr seltene Lokalisation. Hier wurden bisher 9 Fälle publiziert ${ }^{2}$, von denen zwei ein periostales Wachstum aufwiesen ${ }^{4}$

Als periostale, parostale oder auch juxtacorticale Chondrosarkome werden solche Tumore bezeichnet, die sich an der Oberfläche des Knochens bilden und extramedullär wachsen ${ }^{1}$.

In der histologischen Untersuchung der in der zuweisenden Klinik entnommenen Probe zeigte sich der rechts abgebildete, atypisch proliferierende, chondromatöser Tumor mit Zell- und Kernatypien und destruierendem Wachstum im Bezug zum Zungenbein und einer relativ kräftigen kollagenen Pseudokapsel. Es wurde die Diagnose alen (juxtacorticalen) low-grade Chondrosarkoms gestellt.

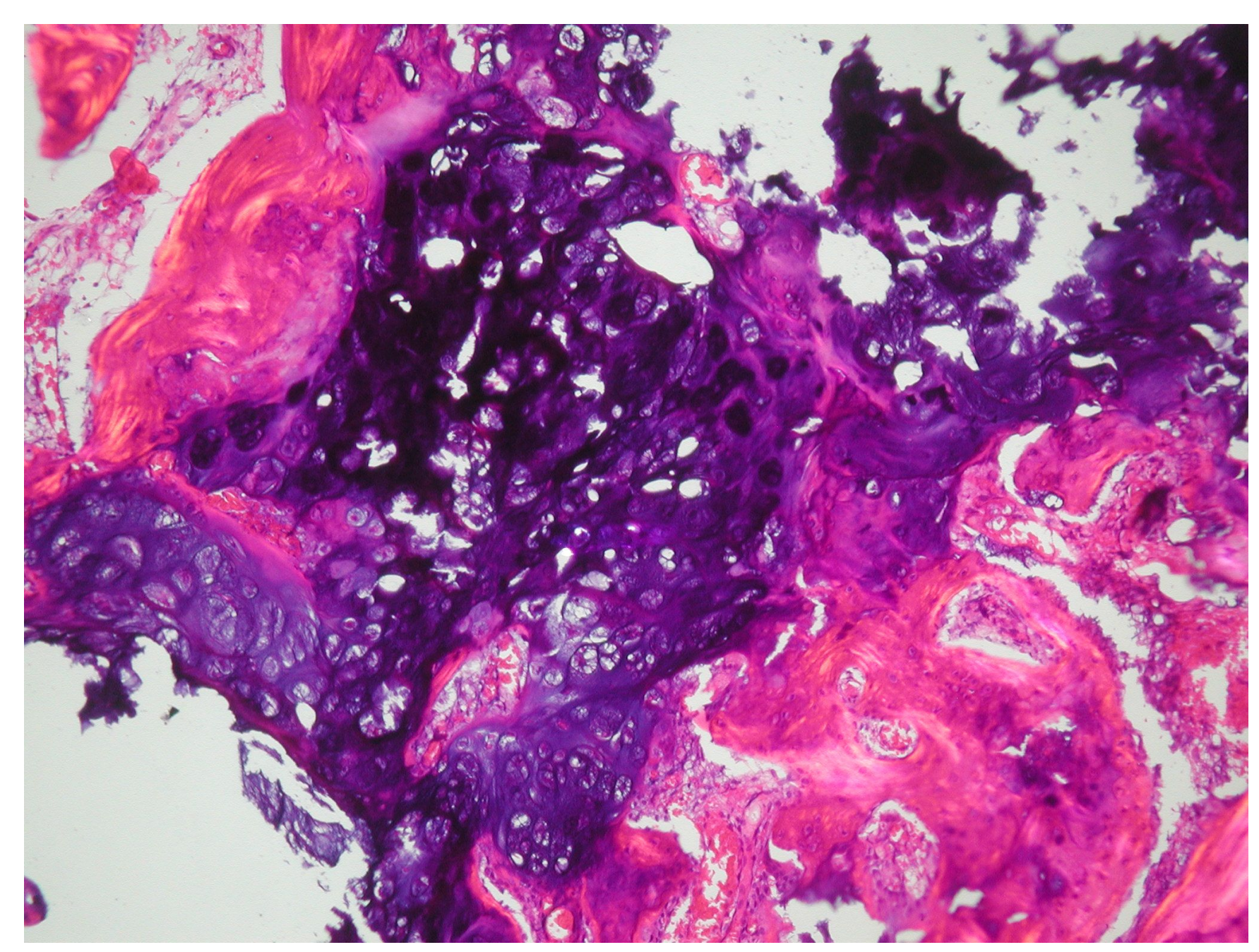

Histologisches Bild des beschriebenen Tumors: Knorpeliges Gewebe (blau) wächst in den Knochen des Zungenbeins (rot) ein.

\section{Abstract:}

Einleitung: Chondrosarkome sind maligne Tumoren, die Chondroid (atypisches Knorpelgewebe) aber in Abgrenzung zum Osteosarkom kein Osteoid bilden. Sie gehen häufiger von Knochen als von Knorpel aus. Betroffen sind in der Regel Becken, Femur, Humerus und Rippen. Selten finden sie sich im Kopf- Halsbereich (10\%) mit Lokalisationen im Bereich der Schädels, Maxilla und des Larynx

Nur 0,1\% aller Kopf-Hals-Malignome entfallen auf Chondrosarkome. In den Halsweichteilen ist die Hauptlokalisation der Kehlkopf mit Hauptbefall des Ringknorpel mit 70\% gegenüber dem Schildknorpel mit 20\% Chondrosarkome des Zungenbeins sind echte Raritäten mit bisher nur 9 publizierten Fällen. Bei dem von uns vorgestellten Fall handelt es sich unseres Wissens erst um den dritten Patienten, bei dem radiologisch ein periostales Wachstum am Zungenbein beschrieben werden konnte.

Kasuistik: Ein erst 30jähriger Patienten stellte sich mit einem bereits histologisch gesichertem low grade Chondrosarkom T1a N0 M0 der linken Halsseite vor. In der MRT-Untersuchung zeigte sich eine 4×5x4 cm große lobulierte, unscharf begrenzte Raumforderung der linken Halsweichteile mit weiter Verdrängung des Hypopharynx nach rechts. Der Tumor konnte in toto unter Mitnahme der Zungenbeinhälfte reseziert werden. Der Pharynx musste nicht eröffnet werden. Es handelt sich um die erste Manifestation bei diesem Patienten. Aussicht: Da diese Tumore kaum auf konventionelle Strahlen- und Chemotherapie ansprechen kommt der vollständigen Resektion eine große Bedeutung zu. Trotz vollständiger Resektion rezidivieren diese Tumore häufig. Rezidive werden bis zu zehn Jahre postoperativ beschrieben. In der onkologischen Nachsorge muss dabei besonderes Augenmerk auf die pulmonale Spätmetastasierung gelegt werden.

\section{Patjent und Ausdehnung}

Klinik

Der 30jährige, männliche Patient in gutem AZ mit ansonsten unauffälliger Anamnese hatte drei Monate zuvor erstmals eine links-cervikale Schwellung bemerkt. In einer auswärtigen Klinik wurde aus dem Tumor eine Biopsie entnommen und das Chondrosarkom diagnostiziert. Wegen der vermuteten Infiltration des Hypopharynx und der A. carotis wurde der Patient uns zur Therapie zugewiesen.

(Medianes Alter bei Erstdiagnose eines Kopf-Hals-Chondrosarkoms ist $56 \mathrm{Jahre}^{1}$ )

Radiologie

Kernspintomografisch wurde eine $4 \times 5 \times 4 \mathrm{~cm}$ messende, lobulierte, teils unscharf vom umliegenden Weichgewebe abgegrenzte Raumforderung beschrieben.

Nach cranial erreichte der Tumor die Zungengrundregion. Nach latero-ventral wurde die Glandula submandibularis verdrängt. Nach medial wuchs die Raumforderung unter Verdrängung des Hypopharynx nach rechts und die

Epiglottisloge wurde radiologisch als infiltriert beschrieben. Nach latero-dorsal reichte der Befund bis an die A. carotis, die in den caudalen Schichten nicht mehr eindeutig abgrenzbar war
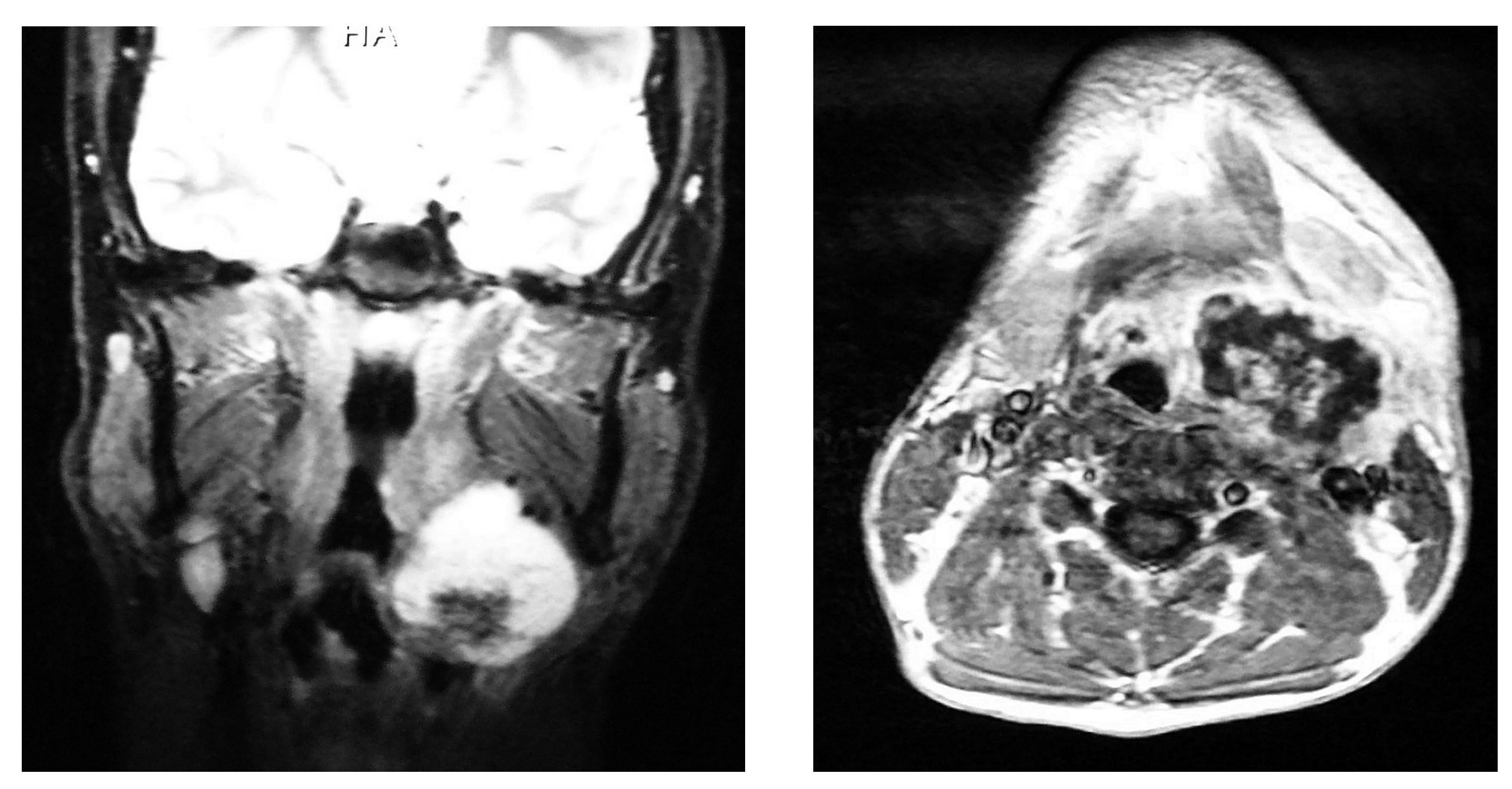

Kernspintomografische Darstellung des Tumors. Beschreibung der Ausdehnung im Text.

\section{Therapie}

Die Therapie des Chondrosarkoms ist primär eine chirurgische. Eine R0-Resektion stellt neben dem Grading den wichtigsten Prognosefaktor dar ${ }^{2,3,7}$. Die Chemotherapie zeigt nur geringe Erfolge, die Bewertung der Strahlentherapie in der Literatur ist widersprüchlich ${ }^{1}$

Wir führten zunächst eine direkte Hypopharyngoskopie durch, bei der sich die Hypopharynxschleimhaut intakt zeigte. Danach erfolgte der offene Zugang unter Einbeziehung der Narbe von der Probengewinnung. Intraoperativ zeigte sich, dass der Tumor ohne Eröffnung des Pharynxschlauches und ohne Verletzung der A. carotis ausgelöst werden konnte. Das Zungenbein war von Tumor umwachsen. Die linke Zungenbeinhälfte wurde mit dem Tumor entfernt, die Tumorkapsel bei der Präparation nicht eröffnet. Die umgebenden Lymphknoten wurden entfernt und zeigten keine Tumorinfiltration (Nach Koch et al. ${ }^{1}$ ist aufgrund aufgrund der geringen Inzidenz regionaler Metastasen von nur 5,6\% ein neck dissection nicht indiziert).

Stadium: pT1, N0, M0 GI-II (low-grade)

Das intraoperative Bild rechts zeigt den lobulierten, von anheftendem Bindegewebe umgebenen Tumor in situ.

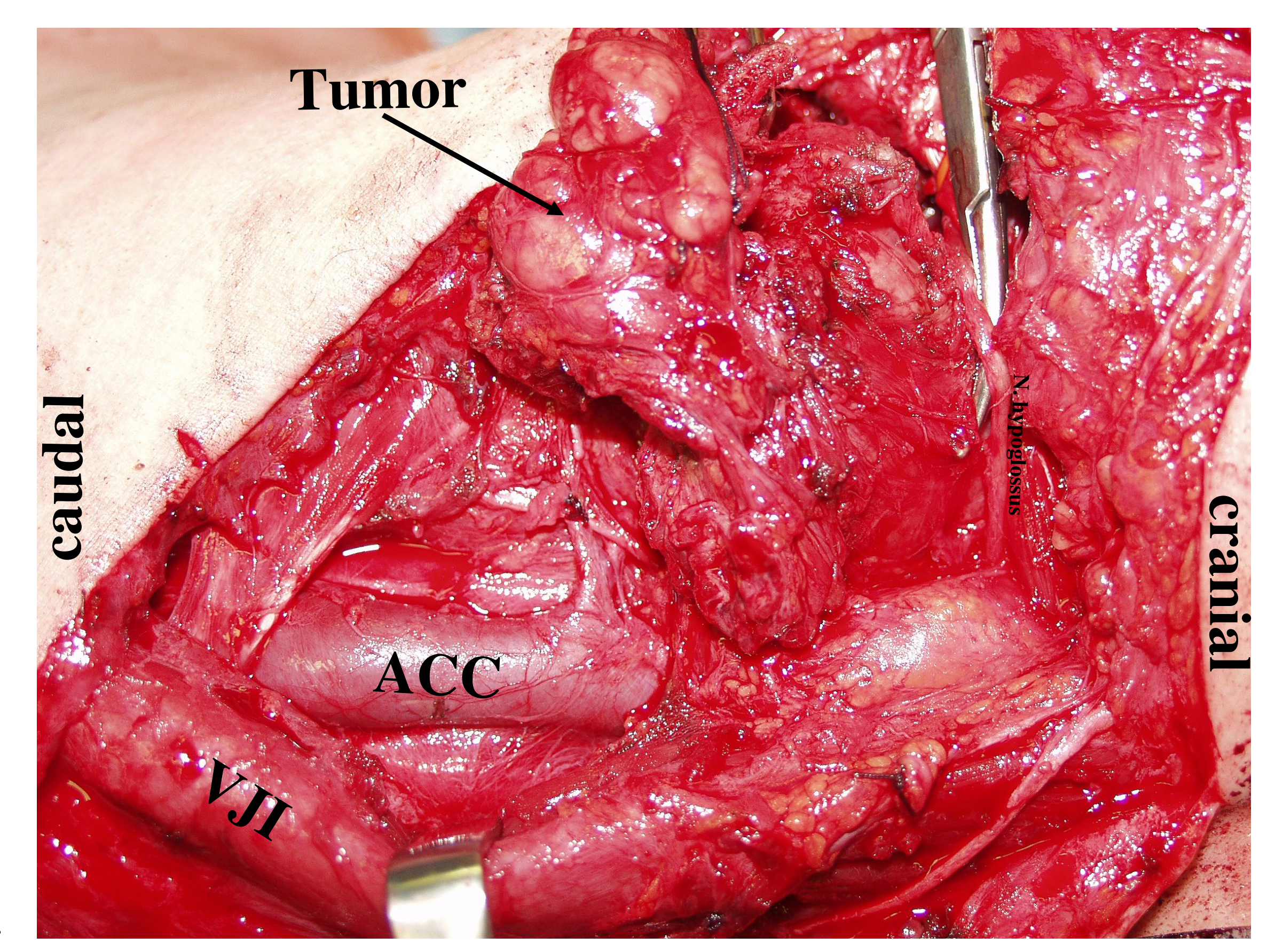

\section{Zusammenfassung}

Das Chondrosarkom des Zungenbeins ist mit bisher 9 publizierten Fällen ausgesprochen selten ${ }^{1,2}$. In Anlehnung an andere Chondrosarkome des Halses steht therapeutisch die vollständige Resektion im Vordergrund. Im vorliegenden Fall eines vergleichsweise jungen Patienten gelang die vollständige Resektion trotz der großen Ausdehnung ohne Verletzung von Pharynx und A. carotis.

Trotz des seltenen Vorkommens dieser Tumore sind Rezidive nach vier ${ }^{4}$ und auch nach acht und zehn ${ }^{6}$ Jahren beschrieben. Entsprechend lang müssen Tumornachsorgeuntersuchungen erfolgen. Besonderes Augenmerk muss dabei auf die pulmonale Spätmetastasierung gelegt werden. 